\title{
On roots of the automorphism group of a circular domain in $\mathbb{C}^{n}$
}

\author{
by JAN M. MYszeWski (Warszawa)
}

\begin{abstract}
We study the properties of the group $\operatorname{Aut}(D)$ of all biholomorphic transformations of a bounded circular domain $D$ in $\mathbb{C}^{n}$ containing the origin. We characterize the set of all possible roots for the Lie algebra of $\operatorname{Aut}(D)$. There exists an $n$-element set $P$ such that any root is of the form $\alpha$ or $-\alpha$ or $\alpha-\beta$ for suitable $\alpha, \beta \in P$.
\end{abstract}

1. Introduction. A bounded domain in $\mathbb{C}^{n}$ is said to be circular if for all $z \in D$ and all $t \in \mathbb{R}, e^{i t} z \in D$ where $i^{2}=-1$.

Given any bounded domain $D \subset \mathbb{C}^{n}$, denote by $\operatorname{Aut}(D)$ the set of all biholomorphic transformations of $D$ onto itself. $\operatorname{Aut}(D)$, when equipped with the compact-open topology, is a locally compact Lie group. A proof of this theorem due to H. Cartan can be found in [4].

In the present paper we study the properties of the Lie algebra of Aut $(D)$. In Section 2 we review some facts on maximal tori of Aut $(D)$. Every finitedimensional complex linear representation of a compact abelian group can be decomposed into a direct sum of one-dimensional subrepresentations (see [1]). To any complex one-dimensional representation of a maximal torus $T$ there corresponds a $\mathbb{C}$-linear functional on the complexification of the Lie algebra of $T$. This functional is called a root of $\operatorname{Aut}(D)$ and is a generalization of that defined for semisimple groups. In Section 3 we characterize the set of all possible roots of $\operatorname{Aut}(D)$. This result generalizes an analogous one obtained by Sunada [5] for $n$-circular domains in $\mathbb{C}^{n}$.

2. Properties of maximal tori of $\operatorname{Aut}(D)$. Assume that $D \subset \mathbb{C}^{n}$ is a bounded circular domain. Let $G$ be the identity component of $\operatorname{Aut}(D)$, denote by 0 the origin of $\mathbb{C}^{n}$ and assume that $0 \in D$. By a theorem of H. Cartan the set $K:=\{f \in G: f(0)=0\}$ is a compact subgroup in $G$

1991 Mathematics Subject Classification: Primary 17B05, 32A07.

Key words and phrases: circular domain, automorphism group, maximal torus, Lie algebra, adjoint representation, root, root subspace. 
and any $f \in K$ is the restriction to $D$ of a $\mathbb{C}$-linear transformation of $\mathbb{C}^{n}$ (see [4]).

A group $T$ is called a torus if it is abelian, connected and compact. For any two maximal tori in $K$ there exists an inner automorphism of $K$ transforming one of them onto the other (see [1], p. 71). The (real) dimension of a maximal torus is called the rank of $K$. We will denote by $T$ any fixed maximal torus in $K$ and by $r$ the rank of $K$.

Let $X$ be any real vector field on $D$. In the standard frame $\partial / \partial z_{1}, \ldots$, $\partial / \partial z_{n}, \partial / \partial \bar{z}_{1}, \ldots, \partial / \partial \bar{z}_{n}$ it can be written in the form

$$
X=\sum_{j=1}^{n} f_{j} \partial / \partial z_{j}+\sum_{j=1}^{n} \bar{f}_{j} \partial / \partial \bar{z}_{j}
$$

$X$ is said to be holomorphic if for any function $h$ holomorphic on $D$ the function $X h$ is holomorphic on $D$. The components $f_{j}, j=1, \ldots, n$, of a real holomorphic vector field (1) are holomorphic on $D$. We say that a real vector field $X$ on $D$ generates a one-parameter group $\left\{g_{t}: t \in \mathbb{R}\right\} \subset G$ if for all $z \in D,\left(g_{t}\right)_{* t=0}(d / d t)=X(z)$.

Denote by $\underline{G}, \underline{K}$ and $\underline{T}$ respectively the Lie algebras of all real vector fields on $D$ generating one-parameter subgroups of $G, K$ and $T$. $\underline{G}, \underline{K}$ and $\underline{T}$ are isomorphic to the Lie algebras of the Lie groups $G, K$ and $T$ respectively (see [3]). Since in the case of circular domains containing the origin we have some information about elements of $\underline{G}, \underline{K}$ and $\underline{T}$ (see Theorem (5)) we operate in $\underline{G}$ rather than in the Lie algebra of the Lie group $G$.

Assume that $D$ is a bounded circular domain in $\mathbb{C}^{n}$ containing the origin. The proof of the following two theorems can be found in [3].

(2) Theorem. Assume that $H$ is an s-dimensional connected compact abelian Lie group (not necessarily maximal) whose elements are restrictions to $D$ of linear transformations of $\mathbb{C}^{n}$. Then $1 \leq s \leq n$ and there exists $a \mathbb{C}$-linear change of coordinates in $\mathbb{C}^{n}$ and real numbers $a_{k}^{j}, j=1, \ldots, s$, $k=s+1, \ldots, n$, satisfying $\sum_{j=1}^{s} a_{k}^{j}=1$ for $k=s+1, \ldots, n$ such that in the new coordinates $w_{1}, \ldots, w_{n}$

(i) for any $h \in H$ there exists an s-tuple $\left(\theta_{1}, \ldots, \theta_{s}\right) \in \mathbb{R}^{s}$ such that the matrix of $h$ is $\operatorname{diag}\left[\exp \left(i \widehat{\theta}_{1}\right), \ldots, \exp \left(i \widehat{\theta}_{n}\right)\right]$ with

$$
\widehat{\theta}_{p}= \begin{cases}\theta_{p}, & 1 \leq p \leq s, \\ \sum_{j=1}^{n} a_{p}^{j} e_{j}, & p>s,\end{cases}
$$

(ii) the vector fields

(2b) $X_{j}=i\left(z_{j} \partial / \partial z_{j}+\sum_{k=s+1}^{n} a_{k}^{j} z_{k} \partial / \partial z_{k}-\bar{z}_{j} \partial / \partial \bar{z}_{j}-\sum_{k=s+1}^{n} a_{k}^{j} \bar{z}_{k} \partial / \partial \bar{z}_{k}\right)$ 
for $j=1, \ldots, s$, form a frame for the Lie algebra $\underline{H}$ of all real vector fields on $D$ generating one-parameter subgroups of $H$.

(3) Theorem. Let $T_{1}$ and $T_{2}$ be any pair of maximal tori in $K=$ $\{f \in \operatorname{Aut}(D): f(0)=0\}$. Assume that $\mathcal{B}_{1}$ and $\mathcal{B}_{2}$ are linear frames in $\mathbb{C}^{n}$ such that the matrices of elements of $T_{1}$ in $\mathcal{B}_{1}$ are of the form $\operatorname{diag}\left[\exp \left(i \widehat{\theta}_{1}\right), \ldots, \exp \left(i \widehat{\theta}_{n}\right)\right]$ with

$$
\widehat{\theta}_{k}= \begin{cases}\theta_{k}, & k \leq r, \\ \sum_{j=1}^{r} a_{k}^{j} \theta_{j}, & k>r,\end{cases}
$$

and the matrices of elements of $T_{2}$ in $\mathcal{B}_{2}$ are of the form $\operatorname{diag}\left[\exp \left(i \widehat{\phi}_{1}\right), \ldots\right.$, $\left.\exp \left(i \widehat{\phi}_{n}\right)\right]$ with

Set

$$
\widehat{\phi}_{k}= \begin{cases}\phi_{k}, & k \leq r, \\ \sum_{j=1}^{r} b_{k}^{j} \phi_{j}, & k>r .\end{cases}
$$

Then

$$
A=\left[\begin{array}{ccc}
a_{r+1}^{1} & \ldots & a_{n}^{1} \\
\ldots \ldots & \ldots & . \\
a_{r+1}^{r} & \ldots & a_{n}^{r}
\end{array}\right], \quad B=\left[\begin{array}{ccc}
b_{r+1}^{1} & \ldots & b_{n}^{1} \\
\ldots \ldots & \ldots & . \\
b_{r+1}^{r} & \ldots & b_{n}^{r}
\end{array}\right] .
$$

(1) $\mathcal{B}_{1}$ and $\mathcal{B}_{2}$ are the same up to the order of elements.

(2) If $C$ is the transition matrix from $\mathcal{B}_{2}$ to $\mathcal{B}_{1}$ then there exists a real $r \times r$ matrix $E$ satisfying the following conditions:

(a) $E\left[\mathbf{1}_{r}, A\right] C=\left[\mathbf{1}_{r}, B\right]$ with $\mathbf{1}_{r}$ the $r \times r$ identity matrix, and $\left[\mathbf{1}_{r}, A\right]$ the $r \times n$ real matrix whose first $r$ columns are those of $\mathbf{1}_{r}$ and the other are those of $A$.

(b) If $v=[1, \ldots, 1] \in \mathbb{R}^{r}$ then $v E=v$.

(3) If $\mathcal{B}_{1}=\mathcal{B}_{2}$, then $A=B$.

(4) Theorem. In the notation of Theorem (2) the map

$$
[\mathbb{R} / 2 \pi \mathbb{Z}]^{s} \ni\left(\theta_{1}, \ldots, \theta_{s}\right) \rightarrow \phi=\operatorname{diag}\left[\exp \left(i \widehat{\theta}_{1}\right), \ldots, \exp \left(i \widehat{\theta}_{n}\right)\right]
$$

is a homomorphism of groups if and only if $a_{k}^{j}$ is an integer for all $j=$ $1, \ldots, s, k=s+1, \ldots, n$.

Proof. For any $k \in\{1, \ldots, n\}$ the map $\mathbb{R}^{s} \ni\left(\theta_{1}, \ldots, \theta_{s}\right) \rightarrow$ $\widehat{\theta}_{k}\left(\theta_{1}, \ldots, \theta_{s}\right) \in \mathbb{R}$ is linear. One can easily check that $\left\{\left(\theta_{1}, \ldots, \theta_{s}\right) \in \mathbb{R}^{s}\right.$ : $\widehat{\theta}_{k}\left(\theta_{1}, \ldots, \theta_{s}\right)=0 \bmod 2 \pi$ for $\left.k=1, \ldots, n\right\}=\left\{\left(\theta_{1}, \ldots, \theta_{s}\right) \in \mathbb{R}^{s}: \theta_{j}=\right.$ $0 \bmod 2 \pi$ for $j=1, \ldots, s\}$ if and only if for any $\left(m_{1}, \ldots, m_{s}\right) \in \mathbb{Z}^{s}$ and for any $k=s+1, \ldots, n, \sum_{j=1}^{s} a_{k}^{j} m_{j} \in \mathbb{Z}$. This is equivalent to the condition $a_{k}^{j} \in \mathbb{Z}$ for $j=1, \ldots, s, k=s+1, \ldots, n$.

3. Properties of the set of roots of the algebra $G$. It can be checked that the Lie algebra $\underline{G}$ of all real vector fields on $D$ generating one- 
parameter subgroups in $\operatorname{Aut}(D)$ is real, i.e. for any nonzero $X$ in $\underline{G}, i X$ is not in $\underline{G}$ (see for instance [3]). It is easy to see that for any $X \in \underline{G}$ the map $\underline{G} \ni Y \rightarrow \operatorname{ad}(X) Y=[X, Y] \in \underline{G}$ is linear.

Denote by $\underline{G}^{c}, \underline{K}^{c}$ and $\underline{T}^{c}$ the complexifications of the algebras $\underline{G}, \underline{K}$ and $\underline{T}$ respectively. In a natural way the map $\underline{G} \times \underline{G} \ni(X, Y) \rightarrow \operatorname{ad}(X) Y$ extends to a $\mathbb{C}$-bilinear map $\underline{G} \times \underline{G} \ni(X, Y) \rightarrow \operatorname{ad}(X) Y \in \underline{G}^{c}$. Denote by $J$ the real vector field on $D$ generating the one-parameter group $\left\{\exp (i t) \operatorname{id}_{D}: t \in \mathbb{R}\right\}$. One easily checks that in the standard frame on $\mathbb{C}^{n}$

$$
J=i\left(\sum_{k=1}^{n} z_{k} \partial / \partial z_{k}-\sum_{k=1}^{n} \bar{z}_{k} \partial / \partial \bar{z}_{k}\right)
$$

(5) THEOREM. In the above notation

(i) $\underline{K}=\operatorname{ker}\left[\operatorname{ad}(J)^{\prime}\right]$, where' denotes the restriction of a map to $\underline{G}$. If $\underline{P}=\operatorname{ker}\left\{\operatorname{id}^{\prime}+\left[\operatorname{ad}(J)^{\prime}\right]^{2}\right\}$, then $\underline{G}=\underline{K}+\underline{P}$ (direct sum) and $[\underline{K}, \underline{P}] \subset \underline{P}$, $[\underline{P}, \underline{P}] \subset \underline{K}$.

(ii) If $\underline{P}^{+}=\left\{X \in \underline{P}^{c}: \operatorname{ad}(J) X=i X\right\}, \underline{P}^{-}=\left\{X \in \underline{P}^{c}: \operatorname{ad}(J) X=\right.$ $-i X\}$, then $\underline{G}^{c}=\underline{K}^{c}+\underline{P}^{+}+\underline{P}^{-}$(direct sum) and $\left[\underline{P}^{+}, \underline{P}^{+}\right]=\left[\underline{P}^{-}, \underline{P}^{-}\right]=$ $\{0\}$.

(iii) For any $X \in \underline{G}^{c}$ there exists a unique decomposition $X=X^{(0)}+$ $X^{(1)}+X^{(2)}$ such that in the standard frame on $\mathbb{C}^{n}$

$$
X^{(m)}=\sum_{j=1}^{n} p_{j}^{m}(z) \partial / \partial z_{j}+\sum_{j=1}^{n} \overline{p_{j}^{m}(z)} \partial / \partial \bar{z}_{j}, \quad m=0,1,2,
$$

and $p_{j}^{m}$ are homogeneous polynomials in $z_{1}, \ldots, z_{n}$ of degree $m$ for $j=$ $1, \ldots, n$. Moreover, $X^{(0)} \in \underline{P}^{-}, X^{(1)} \in \underline{K}^{c}, X^{(2)} \in \underline{P}^{+}$.

See [2] for a proof.

Since $T$ is abelian, $\underline{T}^{c}$ is commutative. By the Jacobi identity, for all $X, Y \in \underline{T}^{c}, \operatorname{ad}(X) \operatorname{ad}(Y)=\operatorname{ad}(Y) \operatorname{ad}(X)$. A nonzero linear functional $\alpha$ : $\underline{T}^{c} \rightarrow \mathbb{C}$ is said to be a root of the algebra $G$ if there exists a nonzero $Y$ in $\underline{G}^{c}$ such that for all $X \in \underline{T}^{c}, \operatorname{ad}(X) Y=\alpha(X) Y$. Assume that $\alpha$ is a root of $\underline{G}$. Then the set $\underline{G}^{\alpha}=\left\{Y \in \underline{G}^{c}: \operatorname{ad}(X) Y=\alpha(X) Y\right.$, for all $\left.X \in \underline{G}^{c}\right\}$ is a complex linear space and is called the root subspace in $\underline{G}^{c}$ corresponding to the root $\alpha$. For any complex vector space $V \subset \underline{G}^{c}$ we put $\Delta(V)=\{\alpha: \alpha$ is a root of $\left.\underline{G}, \underline{G}^{\alpha} \subset V\right\} ; \Delta:=\Delta\left(\underline{G}^{c}\right)$.

Assume that in the coordinates $z_{1}, \ldots, z_{n}$ in $\mathbb{C}^{n}$ all elements of $T$ have diagonal matrices satisfying the conditions of Theorem (2). It can be shown that the vector fields

(6) $\quad Z_{m}=z_{m} \partial / \partial z_{m}+\sum_{j=1}^{r} a_{j}^{m} z_{j} \partial / \partial z_{j}+\bar{z}_{m} \partial / \partial \bar{z}_{m}+\sum_{j=1}^{r} a_{j}^{m} \bar{z}_{j} \partial / \partial \bar{z}_{j}$ 
for $m=1, \ldots, r$ form a frame of the complex space $\underline{T}^{c}$. Denote by $\alpha_{m}$, $m=1, \ldots, r$, the elements of the dual frame, i.e. the $\mathbb{C}$-linear functionals on $\underline{T}^{c}$ such that $\alpha_{k}\left(Z_{m}\right)=\delta_{k m}$ (Kronecker's delta) for $k, m=1, \ldots, r$.

Since $\underline{G}$ is a real vector space, for any $Z \in \underline{G}^{c}$ there exist unique $X, Y \in \underline{G}$ such that $Z=X+i Y$. Denote by $\sigma$ the map $\underline{G}^{c} \ni X+i Y \rightarrow \sigma(X+i Y)=$ $X-i Y . \sigma$ is called the conjugation in $\underline{G}^{c}$ with respect to the real algebra $\underline{G}$. Below we list some properties of $\sigma$.

(7) LEMMA. In the above notation:

$1^{\circ} \sigma^{2}=\mathrm{id}$.

$2^{\circ} \forall X, Y \in \underline{G}^{c} \forall a, b \in \mathbb{C}, \sigma(a X+b Y)=\bar{a} \sigma(X)+\bar{b} \sigma(Y)$.

$3^{\circ} \forall X, Y \in \underline{G}^{c}, \sigma([X, Y])=[\sigma(X), \sigma(Y)]$.

$4^{\circ} \forall X \in \underline{G}^{c}, X \in \underline{G} \Leftrightarrow \sigma(X)=X$.

$5^{\circ} \sigma\left(\underline{P}^{-}\right)=\underline{P}^{+}, \sigma\left(\underline{P}^{+}\right)=\underline{P}^{-}, \sigma\left(\underline{K}^{c}\right)=\underline{K}^{c}$.

$6^{\circ} \sigma\left(\underline{G}^{\alpha}\right)=\underline{G}^{\beta}$, with $\beta=\overline{\alpha \circ \sigma}$.

Proof. $1^{\circ}$ is obvious. For $2^{\circ}$, let $a, b \in \mathbb{R}, X, Y \in \underline{G}$. Then $\sigma((a+$ $b i)(X+i Y))=\sigma((a X-b Y)+i(b X+a Y))=a X-b Y-i(b X+a Y)=$ $(a-b i) \sigma(X+i Y) . \quad 3^{\circ}$ can be checked by a direct computation similar to that of $2^{\circ} .4^{\circ}$ is obvious. For $5^{\circ}$, we first show that $\sigma\left(\underline{P}^{-}\right) \subset \underline{P}^{+}$. Assume that $X \in \underline{P}^{-}$. Then $\operatorname{ad}(J) \sigma(X)=[J, \sigma(X)]=\sigma([\sigma(J), X])=$ $\sigma([J, X])=\sigma(-i X)=i \sigma(X)$ (we have used the fact that $J \in \underline{T} \subset \underline{G}$ ). Hence $\sigma(X) \in \underline{P}^{+}$. The converse inclusion can be checked in the same way. For $6^{\circ}$ assume that $Y$ is a nonzero element of $\underline{G}^{\alpha}$. Let $X$ be any element of $\underline{T}^{c}$. Then $\operatorname{ad}(X) \sigma(Y)=[X, \sigma(Y)]=\sigma([\sigma(X), Y])=\sigma(\alpha(\sigma(X)) Y)=$ $\overline{\alpha(\sigma(X))} \sigma(Y)$. Hence $\sigma(Y) \in \underline{G}^{\beta}$ with $\beta=\overline{\alpha \circ \sigma}$. In the same way one checks that $\sigma\left(\underline{G}^{\beta}\right) \subset \underline{G}^{\alpha}$. Since $\sigma$ is bijective, this completes the proof.

(8) LEMmA. In the above notation, for any $\alpha \in \Delta$ exactly one of the following conditions holds:
(a) $\underline{G}^{\alpha} \subset \underline{P}^{-}$,
(b) $\underline{G}^{\alpha} \subset \underline{K}^{c}$,
(c) $\underline{G}^{\alpha} \subset \underline{P}^{+}$.

Proof. Let $\alpha \in \Delta$ and let $Y$ be any nonzero element of $\underline{G}^{\alpha}$. By Theorem (5) there are unique $Y^{(0)} \in \underline{P}^{-}, Y^{(1)} \in \underline{K}^{c}, Y^{(2)} \in \underline{P}^{+}$such that $Y=Y^{(0)}+Y^{(1)}+Y^{(2)}$ and $\operatorname{ad}(J) Y=\alpha(J) Y$. On the other hand, $\operatorname{ad}(J) Y=$ $-i Y^{(0)}+0 Y^{(1)}+i Y^{(2)}$. It follows that at most one of the components $Y^{(0)}, Y^{(1)}, Y^{(2)}$ is nonzero.

Assume that in the coordinates $z_{1}, \ldots, z_{n}$ in $\mathbb{C}^{n}$ the matrices of all elements of $T$ have the form $\operatorname{diag}\left[\exp \left(i \widehat{\theta}_{1}\right), \ldots, \exp \left(\widehat{\theta}_{n}\right)\right]$ with $\widehat{\theta}_{k}$ of the form (2a) with $s=r$. Let $\alpha_{k}$ for $k=1, \ldots, r$ be the elements of the dual frame to (6) in $\underline{T}^{c}$ and define

$$
\widehat{\alpha}_{k}=\left\{\begin{array}{ll}
\alpha_{k}, & 1 \leq k \leq r, \\
\sum_{j=1}^{n} a_{k}^{j} \alpha_{j}, & k>r,
\end{array} \quad \text { for } k=1, \ldots, n .\right.
$$


Below we investigate some properties of $\underline{G}$ associated to root subspaces contained in $\underline{P}^{-}, \underline{P}^{+}$and $\underline{K}^{c}$ respectively. $Z_{m}$ are the vector fields defined in (6) for $m=1, \ldots, r$.

(10) LEMMA. In the above notation, if $\alpha \in \Delta\left(\underline{P}^{-}\right)$then

(a) there exists $k \in\{1, \ldots, n\}$ such that $\alpha=-\widehat{\alpha}_{k}$.

(b) $\underline{G}^{\alpha} \subset \sum_{j, \hat{\theta}_{j}=\hat{\theta}_{k}} \mathbb{C} \partial / \partial z_{j}+\sum_{j, \hat{\theta}_{j}=\hat{\theta}_{k}} \mathbb{C} \partial / \partial \bar{z}_{j}$ (direct sum of one-dimensional subspaces), where both sums are over all $j \in\{1, \ldots, n\}$ such that $\widehat{\theta}_{j}=\widehat{\theta}_{k}$.

Proof. Let $Y$ be a nonzero element of $G^{\alpha}$. By Theorem (5) there exist $y_{j}, y_{j}^{\prime} \in \mathbb{C}$ for $j=1, \ldots, n$ such that $Y=\sum_{j=1}^{n} y_{j} \partial / \partial z_{j}+\sum_{j=1}^{n} y_{j}^{\prime} \partial / \partial \bar{z}_{j}$. Let $X=\sum_{j=1}^{r} x_{j} Z_{j}$ be an arbitrary element of $\underline{T}^{c}$ with $x_{j} \in \mathbb{C}$ for $j=1, \ldots, r$. Assume that $\alpha=\sum_{j=1}^{r} b_{j} \alpha_{j}$ with $b_{j} \in \mathbb{C}$ for $j=1, \ldots, r$. By a direct computation one finds that $\operatorname{ad}(X) Y=\alpha(X) Y$ if and only if

$$
\begin{gathered}
y_{m}\left[\sum_{j=1}^{r}\left(b_{j}+\delta_{j m}\right) x_{j}\right]=y_{m}^{\prime}\left[\sum_{j=1}^{r}\left(b_{j}+\delta_{j m}\right) x_{j}\right]=0 \quad \text { for } m=1, \ldots, r, \\
y_{m}\left[\sum_{j=1}^{r}\left(b_{j}+a_{j}^{m}\right) x_{j}\right]=y_{m}^{\prime}\left[\sum_{j=1}^{r}\left(b_{k}+a_{j}^{m}\right) x_{j}\right]=0 \quad \text { for } m=r+1, \ldots, n .
\end{gathered}
$$

The above equations are satisfied for any $X$ in $\underline{T}^{c}$ if and only if $y_{m}\left(\alpha+\widehat{\alpha}_{m}\right)=$ $y_{m}^{\prime}\left(\alpha+\widehat{\alpha}_{m}\right)=0$ for $m=1, \ldots, n$. Since $Y \neq 0$, there exists $k \in\{1, \ldots, n\}$ such that $y_{k} \neq 0$ or $y_{k}^{\prime} \neq 0$. Hence $\alpha+\widehat{\alpha}_{k}=0$ and $y_{m}=y_{m}^{\prime}=0$ for $m \in\{1, \ldots, n\}$ such that $\widehat{\alpha}_{m} \neq \widehat{\alpha}_{k}$ or equivalently such that $\widehat{\theta}_{m} \neq \widehat{\theta}_{k}$.

(11) LEMMA. In the above notation, if $\alpha \in \Delta\left(\underline{K}^{c}\right)$ then

(a) there exist $p, q \in\{1, \ldots, n\}$ such that $\alpha=\widehat{\alpha}_{p}-\widehat{\alpha}_{q}$,

(b) $\underline{G}^{\alpha} \subset \sum_{j, k, \hat{\theta}_{j}-\hat{\theta}_{k}=\hat{\theta}_{p}-\hat{\theta}_{q}} \mathbb{C} z_{j} \partial / \partial z_{k}+\sum_{j, k, \hat{\theta}_{j}-\hat{\theta}_{k}=\hat{\theta}_{p}-\hat{\theta}_{q}} \mathbb{C} \bar{z}_{j} \partial / \partial \bar{z}_{k}(d i-$ rect sum of one-dimensional subspaces).

P r o of. Let $Y=\sum_{j, k=1}^{n} y_{j k} z_{j} \partial / \partial z_{k}+\sum_{j, k=1}^{n} y_{j k}^{\prime} \bar{z}_{j} \partial / \partial \bar{z}_{k}$ be any nonzero element of $\underline{G}^{\alpha}$, let $X=\sum_{j=1}^{n} x_{j} Z_{j}$ be an arbitrary element of $\underline{T}^{c}$, and assume that $\alpha=\sum_{j=1}^{r} b_{j} \alpha_{j}$. By a direct computation one finds that $\operatorname{ad}(X) Y=\alpha(X) Y$ for all $X \in \underline{T}^{c}$ if and only if $y_{k m}\left[\widehat{\alpha}_{m}-\widehat{\alpha}_{k}+\alpha\right]=$ $y_{k m}^{\prime}\left[\widehat{\alpha}_{m}-\widehat{\alpha}_{k}+\alpha\right]=0$ for all $k, m \in\{1, \ldots, n\}$. A reasoning similar to that in the proof of Lemma (10) completes the proof.

(12) LEMMA. In the above notation, if $\alpha \in \Delta\left(\underline{P}^{+}\right)$then

(a) there exists $p \in\{1, \ldots, n\}$ such that $\alpha=\widehat{\alpha}_{p}$,

(b) $\underline{G}^{\alpha} \subset \sum_{j, k, l, \hat{\theta}_{j}+\hat{\theta}_{k}-\hat{\theta}_{l}=\hat{\theta}_{p}} \mathbb{C} z_{j} z_{k} \partial / \partial z_{l}+\sum_{j, k, l, \hat{\theta}_{j}+\hat{\theta}_{k}-\hat{\theta}_{l}=\hat{\theta}_{p}} \mathbb{C} \bar{z}_{j} \bar{z}_{k} \partial / \partial \bar{z}_{l}$ (direct sum of one-dimensional subspaces). 
Proof. Let $Y=\sum_{j, k, l=1}^{n} y_{j k l} z_{j} z_{k} \partial / \partial z_{l}+\sum_{j, k, l=1}^{n} y_{j k l}^{\prime} \bar{z}_{j} \bar{z}_{k} \partial / \partial \bar{z}_{l}$ be a nonzero element of $\underline{G}^{\alpha}$ and let $X=\sum_{j=1}^{n} x_{j} Z_{j}$ be an arbitrary element of $\underline{T}^{c}$. Assume that $\alpha=\sum_{j=1}^{r} b_{j} \alpha_{j}$. By a direct computation one finds that $\operatorname{ad}(X) Y=\alpha(X) Y$ for all $X \in \underline{T}^{c}$ if and only if $y_{j k l}\left[\widehat{\alpha}_{l}-\widehat{\alpha}_{j}-\widehat{\alpha}_{k}+\alpha\right]=$ $y_{j k l}^{\prime}\left[\widehat{\alpha}_{l}-\widehat{\alpha}_{j}-\widehat{\alpha}_{k}+\alpha\right]=0$ for $j, k, l \in\{1, \ldots, n\}$. Since $Y \neq 0$, there exists a triple $(p, q, s) \in\{1, \ldots, n\}^{3}$ such that $y_{p q s} \neq 0$ or $y_{p q s}^{\prime} \neq 0$. Hence $\alpha=\widehat{\alpha}_{p}+\widehat{\alpha}_{q}-\widehat{\alpha}_{s}$ and $y_{j k l}=y_{j k l}^{\prime}=0$ for all $j, k, l \in\{1, \ldots, n\}$ such that $\widehat{\theta}_{j}+\widehat{\theta}_{k}-\widehat{\theta}_{l} \neq \widehat{\theta}_{p}+\widehat{\theta}_{q}-\widehat{\theta}_{s}$.

On the other hand, by Lemma $7\left(6^{\circ}\right), \sigma\left(\underline{G}^{\alpha}\right)=\underline{G^{\beta}}$ with $\beta=\overline{\alpha \circ \sigma}$. Let $X=X^{\prime}+i X^{\prime \prime}$ with $X^{\prime}, X^{\prime \prime} \in \underline{T}$. Then $\beta(\bar{X})=\overline{\alpha\left(X^{\prime}-i X^{\prime \prime}\right)}=\overline{\alpha\left(X^{\prime}\right)}+$ $i \overline{\alpha\left(X^{\prime \prime}\right)}$. Since for any $\alpha \in \Delta\left(\underline{P}^{-}\right), \alpha=\sum_{j} b_{j} \alpha_{j}$ with $b_{j}$ real for $j=1, \ldots, r$ and $\alpha(\underline{T}) \subset i \mathbb{R}$, we have $\beta=-\alpha$. Hence there exists $p \in\{1, \ldots, n\}$ such that $\alpha=\widehat{\alpha}_{p}$ and $y_{j k l}=y_{j k l}^{\prime}=0$ for $j, k, l \in\{1, \ldots, n\}$ such that $\widehat{\theta}_{j}+\widehat{\theta}_{k}-\widehat{\theta}_{l} \neq \widehat{\theta}_{p}$.

(13) Corollary. The assertion $6^{\circ}$ in Lemma (7) can be formulated as follows: $\sigma\left(\underline{G}^{\alpha}\right)=\underline{G}^{-\alpha}$.

(14) Theorem. Let $D$ be a bounded circular domain in $\mathbb{C}^{n}$ containing the origin, and let $T$ be any maximal torus in $\operatorname{Aut}(D)$. Let $\underline{G}, \underline{T}$ be the Lie algebras of real vector fields generating all one-parameter subgroups in $\operatorname{Aut}(D)$ and $T$ respectively. Let $\Delta$ be the set of all roots of $\underline{G}$. Then

(i) For any $\alpha \in \Delta$ we have $-\alpha \in \Delta$.

(ii) There exists a set $\Pi$ in the dual space to $\underline{T}^{c}$ with the following properties:

(a) $\Pi$ has at most $n$ elements.

(b) For any $\alpha \in \Delta$ one of the following holds:

$$
\alpha=\beta, \quad \alpha=-\beta, \quad \alpha=\beta-\gamma \quad \text { for some } \beta, \gamma \in \Pi .
$$

(iii) If in the coordinates $z_{1}, \ldots, z_{n}$ in $\mathbb{C}^{n}$ all elements of $T$ have diagonal matrices satisfying the conditions of Theorem (2), then $\Pi=\left\{\widehat{\alpha}_{k}: k=\right.$ $1, \ldots, n\}$ with $\widehat{\alpha}_{k}$ of the form (9).

Proof. This is an easy consequence of Lemmas 8, 10, 11, 12.

(15) Remark. For $r=n$ we obtain the $n$-circular case studied by T. Sunada [5].

\section{References}

[1] J. F. Adams, Lectures on Lie Groups, Benjamin, New York 1969.

[2] W. Kaup and H. Upmeier, Banach spaces with biholomorphically equivalent balls are isomorphic, Proc. Amer. Math. Soc. 58 (1976), 129-133. 
[3] J. M. Myszewski, On maximal tori of the automorphism group of circular domain in $\mathbb{C}^{n}$, Demonstratio Math. 22 (4) (1989), 1067-1080.

[4] R. Narasimhan, Several Complex Variables, Chicago Lectures in Mathematics, The University of Chicago Press, Chicago \& London 1971.

[5] T. Sunada, Holomorphic equivalence problem for bounded Reinhardt domains, Math. Ann. 235 (1978), 111-128.

INSTITUTE OF APPLICATIONS OF MATHEMATICS

SGGW-ACADEMY OF AGRICULTURE

NOWOURSYNOWSKA 166

02-975 WARSZAWA, POLAND

Reçu par la Rédaction le 14.9.1990

Révisé le 10.1.1991 